\title{
Testing strategies for the safety of nanoparticles used in medical applications
}

\begin{abstract}
"A better understanding of how properties of NPs define their interactions with cells, tissues and organs in exposed humans is a considerable scientific challenge, but one that must be addressed if there is to be safe and responsible use of biomedical NPs."
\end{abstract}

Nanoparticles (NPs) have unique physicochemical properties that are different from those of the bulk material. Properties, such as small size $(<100 \mathrm{~nm})$, relatively high surfaceto-volume ratio, quantum dot effect and reactivity, allow a wide range of different applications in modern industry. Nanotechnology is considered as a technology of the future, with great potential in biomedical applications. It is becoming increasingly important in nanodiagnostics (medical imaging, biosensors, contrast agents and in vitro 'lab-on-a-chip'), drug-delivery devices (for gene delivery and targeting drugs) and regenerative medicine (prostheses and implants). NPs have already been used for treating a variety of human diseases including infections, genetic disorders and tumors.

The unique properties of NPs, whilst likely to benefit many aspects of our lives, are also a cause of concern as their possible impact on human health is not known. With some NP-based products already in use in nanomedicine and many more under development, it is critically important that the potential risks are properly assessed. Nanomedicine brings humans into direct contact with NPs and it is therefore essential for both public confidence and nanotechnology companies that appropriate risk assessments are undertaken in relation to health and safety. Clearly, there is a pressing need to understand how engineered NPs interact with the human body following exposure of patients or consumers, in the workplace or from the environment. The European Commission has adopted the Action Plan 'Nanosciences and nanotechnologies: an action plan for Europe 2005-2009' [101], defining actions for the 'immediate implementation of a safe, integrated and responsible strategy for nanosciences and nanotechnologies'. In addition, there are ongoing discussions within European Commission committees [102], and within REACH (the Registration, Evaluation, Authorization and Restriction of Chemicals)-competent authorities and the subgroup on nanomaterials as to hazard and risk assessment of nanomaterials [103,104], although originally, nanotechnologies and nanomaterials were not clearly included in the scope of REACH.

With respect to human health, the current methodologies described in the Technical Guidance Documents [105] are generally likely to be able to identify the hazards associated with the use of NPs. However, the specific features and properties of NPs that may contribute to their potential toxicity are not fully addressed. Special attention should be given to the NPs' physicochemical characteristics and the changes that may occur under local environmental conditions. Such properties and their changes include size, surface, chemical composition, bioavailability, solubility, agglomeration, dissociation and adsorption of environmental substances, all of which may have an impact on the ultimate toxicity of the NPs.

One of the main problems encountered in testing NPs for human toxicity has been the lack of appropriate standardized protocols. Expressing the NP dose as a mass concentration is probably not the best description for the determination of the dose-response relationship (as it is for conventional chemicals or drugs). Other metrics such as number of NPs or surface area are likely to be more appropriate. With respect to exposure to NPs, the validity and appropriateness of existing technologies are not always clear. The environmental effects of NPs need to be evaluated through the establishment of scenarios reflecting their typical production and use. Exposure and dose-effect models may need to be adapted, taking into account their changing physicochemical properties over time, and their slow degradation.

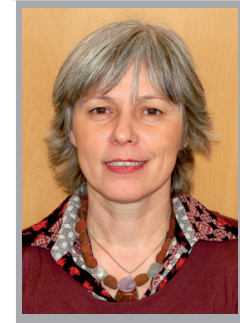

Maria Dusinska \&

NanoTEST consortium*

${ }^{+}$Author for correspondence:

Health Effects Group,

Norwegian Institute for Air

Research (NILU), Centre for

Ecology and Economics,

POB 100, Instituttvn. 18,

N-2027 Kjeller, Norway

Tel.: +47 6389 8157;

Fax: +476389 8050;

maria.dusinska@nilu.no

*NanoTEST consortium:

M Dusinska, LM Fjellsbø,

Z Magdolenova, A Rinna,

E Runden Pran, A Bartonova,

ES Heimstad, M Harju, L Tran,

B Ross, L Juillerat,

B Halamoda Kenzaui,

F Marano, S Boland,

$R$ Guadaginini, M Saunders,

L Cartwright, S Carreira,

M Whelan, CH Kelin, A Worth

T Palosaari, E Burello,

C Housiadas, M Pilou,

K Volkovova, J Tulinska,

A Kazimirova, M Barancokova,

K Sebekova, M Hurbankova,

Z Kovacikova, L Knudsen,

MS Poulsen, $T$ Mose, M Vilà,

L Gombau, B Fernandez,

J Castell, A Marcomini,

G Pojana, D Bilanicova

\& D Vallotto

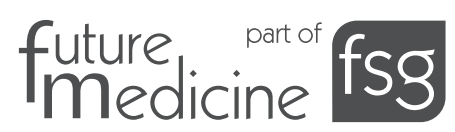


Improvements to the methodologies should take into account the following factors:

- Tests for exposure should be designed that express dose in terms of number concentration and surface area, rather than mass concentration;

- NPs may agglomerate and disagglomerate in different environments, which may affect their properties and these need to be characterized;

- Impurities within, and adsorbed species on the surface of, NPs may have significant effects on risks and these possibilities should be taken into account;

- Biological processes involving NPs, including translocation, cellular uptake and toxicological mechanisms, are still largely unknown and testing methodologies have to address these possible pathways of effect.

It should also be noted that reference materials for the evaluation of NPs have not yet been identified. With respect to specific concrete suggestions, there is a clear need for validated in vitro assays for NP evaluation. In vitro tests should address key properties of the NPs, their biopersistence and possible mechanisms such as free-radical generation, cellular toxicity, cell activation, genotoxicity and other generic end points. In vitro tests should also provide target cell-specific end points addressing specific organs or tissues such as effects on nerve cells or the phagocytic capacity of macrophages.

\section{"With some NP-based products already in use in nanomedicine and many more under development, it is critically important that the potential risks are properly assessed."}

The FP7 project NanoTEST [106] addresses these requirements in relation to the toxicological profile of NPs used in medical diagnostics. The overall aim of this project is to develop alternative testing strategies and high-throughput toxicity testing protocols using in vitro and in silico methods that are essential for the risk assessment of these NPs. To be able to achieve this ambitious goal, the specific aims of NanoTEST are:

- To carry out a detailed characterization of selected NPs, in order to define their main physicochemical properties;

- To study specific and nonspecific interactions of NPs with molecules, cells and organs and to develop in vitro methods that can identify the toxicological potential of NPs;
- To validate in vitro findings in short-term in vivo models, to study manifestation of particle effects in animals and humans, and to assess individual susceptibility in the response to NPs;

- To perform both structure-activity modeling and physiologically based pharmacokinetic modeling of NPs;

- To adapt the most advanced and promising assays for high-throughput automated systems;

- To prepare for validation by the European Centre for the Validation of Alternative Methods (ECVAM).

All pertinent physicochemical properties of concern, for example size distribution, shape, specific surface area, porosity, chemical composition, purity, impurities of concern, surface chemistry, surface charge and crystal structure are being determined in order to ensure high-quality results. Dispersion and stability of selected NPs in aqueous and biological media, as well as their interactions with culture media components, are also being investigated. Eventually, specific analytical protocols will be developed for the quantitative determination of the uptake and distribution of selected NPs in biological tissues after in vitro and in vivo exposure experiments.

This project will address many key elements in the evaluation of NP exposure, cellular uptake, transport and toxicological effects using different biological systems. The mechanism of action of particles is likely to involve oxidative stress and inflammation. We will therefore focus on the cross-cutting areas of cellular toxicity, oxidative stress, inflammation, immunotoxicity, genotoxicity and related end points. We aim to propose relevant in vitro toxicological models based on reference cell lines of these organs, in order to identify reference biological markers and end points that can be used to test the possible toxicity of medical NPs. To identify these biomarkers, we will utilize different cell cultures (in vitro models), organotypic cell culture and small fragments of organs (ex vivo models), exposing them to NPs in clinical use. This will allow us to determine the mechanism of action and to predict the biological and physiological response of exposure to medical NPs and finally to evaluate the potential health hazards of NPs. Whilst it is not possible to consider all organs that may potentially be affected by exposure to medical NPs, we intend to use a range of representative organs and to define representative 
cell lines of these organs. We will focus on cells derived from eight different biological target systems: blood, vascular, liver, kidney, lung, placenta, digestive and CNS. All in vitro studies will be coordinated and harmonized as much as possible by using the same batch of NPs, handling and safety procedures, exposure time, concentration and protocols with similar end points, in order to be able to compare results and to evaluate the most relevant biomarkers and target cell types. Experimental protocols (standard operating procedures) for biomarker detection to be used across the cell systems are being developed. The toxicological profile of NPs in vitro will be validated in an experimental in vivo model addressing the same toxicological end points and organs.

\section{"Nanotechnology is considered as a technology of the future, with great potential in biomedical applications."}

A better understanding of how properties of NPs define their interactions with cells, tissues and organs in exposed humans is a considerable scientific challenge, but one that must be addressed if there is to be safe and responsible use of biomedical NPs. NanoTEST will evaluate toxic effects and interactions of NPs used in nanomedicine. Testing strategy and a battery of in vitro and in silico assays to evaluate the toxic effects of NPs used in nanomedicine will be developed, but these methodologies, we hope, have a broader application to the safety of other nanomaterials. Following development of standard operating procedures and generation of a common database, and in parallel with in silico assays (quantitative structure activity relationship and physiologically based pharmacokinetic modeling), NanoTEST will evaluate the toxic effects and interactions of NPs used in nanomedicine. Results will be validated in an experimental, ethically approved in vivo model. The most advanced and standardized techniques will be adapted for automation and prepared for validation by the Joint Research Centre (ECVAM). Finally, we will propose recommendations for evaluating the potential risks associated with new medical NPs, which will be communicated to the scientific and industrial community.

\section{Financial \& competing interests disclosure}

The NanoTEST consortium is supported by the European Commission FP7 (Health-2007-2001.3-4), contract no: 201335. The NanTEST consortium also receives contributions from Norwegian Institute for Air Research (NILU), Kjeller, Norway; Institute of Occupational Medicine (IOM), UK; Centre Hospitalier Universitaire Vaudois (HospicesCHUV), Switzerland; University Paris Diderot Parts7 (UP7), France; University Hospitals Bristol NHS Foundation Trust (UBHT), UK; Commission of the European Communities - Directorate General Joint Research Centre (JRC), Italy; National Centre for Scientific Research Demokritos (DEMOKRITOS), Greece; Slovak Medical University (SMU), Slovakia; University of Copenhagen (UCPH), Denmark; Advanced In Vitro Cell Technolgoies SL (ADVANCELL), Spain; and Università Ca' Foscari di Venezia (University of Venice) (UNIVE), Italy.

The authors have no other relevant affiliations or financial involvement with any organization or entity with a financial interest in or financial conflict with the subject matter or materials discussed in the manuscript apart from those disclosed.

No writing assistance was utilized in the production of this manuscript.

\section{Websites}

101 European Commission (2004). Towards a European strategy for nanotechnology. http://cordis.europa.eu/nanotechnology/ actionplan.htm

102 European Commission (2007). SCENIHR (EU Scientific Committee on Emerging and Newly Identified Health Risks) report 'Opinion on the appropriateness of the risk assessment methodology in accordance with the technical guidance documents for new and existing substances for assessing the risks of nanomaterials'. http://ec.europa.eu/health/ph_risk/ committees/04_scenihr/docs/ scenihr_o_004c.pdf

103 European Parliament: Regulation (EC) no 1907/2006 of the European Parliament and of the council of 18 December 2006 concerning the registration, evaluation, authorisation and restriction of chemicals (REACH), (OJ L 396, 30.12.2006) (2006).

http://eur-lex.europa.eu/LexUriServ/ LexUriServ.do?uri=OJ:L:2006:396:SOM:en: HTML
104 European Commission (2008). Follow-up to the 6th meeting of the REACH competent authorities for the implementation of regulation (EC) 1907/2006; (REACH). http://ec.europa.eu/environment/chemicals/ reach/pdf/nanomaterials.pdf

105 Technical Guidance Documents. http://guidance.echa.europa.eu/guidance_ en.htm

106 NanoTEST.

www.nanotest-fp7.eu 